\title{
QUADRATIC FORMS OF HEIGHT TWO
}

BY

ROBERT W. FITZGERALD

\begin{abstract}
Quadratic forms of height two and leading form defined over the base field are determined over several fields. Also forms of height and degree two over an arbitrary field are classified.
\end{abstract}

Knebusch, in his theory of generic splitting fields, defines the height of a quadratic form $q$ over a field $F$ of characteristic not 2. A form $q$ has height 1 if $q$ splits over $F(q)$, where $F(q)$ denotes the function field of the projective variety $q=0$, and a form $q$ has height $n>1$ if, over $F(q), q$ has height $n-1$.

The (even-dimensional) forms of height 1 are Pfister forms and their scalar multiples. This is a restatement of the well-known fact that Pfister forms are the only (anisotropic) strongly multiplicative forms. We consider here forms of height 2 and obtain a classification in several cases. Forms of height 2 are of interest because they are the simplest possible forms aside from Pfister forms. Nonetheless, such forms remain somewhat mysterious, and a complete classification appears to be extremely difficult.

This paper extends Knebusch's work, particularly the last two sections of [13]. From the remarks above, we have that (even-dimensional) $q$ has height 2 if and only if $\operatorname{ker}(q \otimes F(q))$ is a scalar times some Pfister form $\rho$. The degree of $q$ is $n$ if $\rho$ is an $n$-fold Pfister form (or 0 if $q$ is odd dimensional). Particular attention is given to the case where $\rho$ is defined over $F-q$ is then called a good form. Knebusch has shown any form of degree 1 and height 2 is good and has classified these. However, height 2 forms need not in general be good.

$\S 1$ studies good forms of height 2. Such forms of degrees 0 and 2 are classified. By placing relatively strong restrictions on $F$, all good forms of height 2 may be determined. In particular, there is a classification for fields of transcendence degree $\leqslant 4$ over $\mathbf{C}$, transcendence degree $\leqslant 2$ over $\mathbf{R}$, global fields and $Q((t))$.

$\$ 2$ considers forms of height and degree 2 . Although we do not obtain a complete description (except in a few cases), such forms are shown to be one of five types. This is sufficient for some applications. For example, if $q$ has height and degree 2 then the Witt Kernel $W(F(q) / F)$ is a Pfister ideal.

Notation and terminology will be taken from [15], except we will write $\operatorname{ker}(q)$ for the anisotropic part of a form $q$. The discriminant of $q$ will be denoted by $d(q)$; thus $d(q)=(-1)^{n(n-1) / 2} \operatorname{det}(q)$, where $n=\operatorname{dim} q$.

Received by the editors April 11, 1983 and, in revised form, August 11, 1983.

1980 Mathematics Subject Classification. Primary 10C05; Secondary 13K05.

(1984 American Mathematical Society $0025-5726 / 84 \$ 1.00+\$ .25$ per page 
$P F\left[P_{n} F\right]$ denotes the set of $F$-Pfister forms [resp. $n$-fold $F$-Pfister forms]. $G P F$ denotes the set of forms similar to an $F$-Pfister form; similarly for $G P_{n} F$. We denote the pure part of a Pfister form $\rho$ by $\rho^{\prime}$. That is, $\rho^{\prime}$ is the uniquely determined subform $\rho$ such that $\rho \simeq \rho^{\prime} \perp\langle 1\rangle$. A form $q$ is a Pfister neighbor if there is a $\rho \in G P F$ and a form $\eta$ such that $\rho \simeq q \perp \eta$ and $\operatorname{dim} q>\operatorname{dim} \eta$. The form $\eta$ is the complementary form of $q$.

An ideal $A$ of the Witt ring $W F$ of $F$ is a Pfister ideal it is is generated by Pfister forms. It is a $\left\{n_{1}, \ldots, n_{k}\right\}$-Pfister ideal if the generators can be taken as $n_{i}$-fold Pfister forms, $1 \leqslant i \leqslant k$. The ideal $A$ is a strong $n$-Pfister ideal if each anisotropic form in $A$ is isometric to a sum of multiplies of $n$-fold Pfister forms in $A$.

Throughout the paper we will use the terminology of Knebusch for function fields, generic splitting towers, etc.; see $[12,13]$. In particular, excellent forms are defined inductively by: All forms of dimension $\leqslant 1$ are excellent. A form $q$ of dimension $\geqslant 2$ is excellent if $q$ is a Pfister neighbor and the complementary form of $q$ is excellent. Let $W(F(q) / F)$ denote the kernel of the homomorphism of Witt rings $W F \rightarrow W F(q)$ induced by the field extension $F(q) / F$. The ideal $J_{n} F=\{q \in$ $W f \mid \operatorname{deg} q \geqslant n\}$. For a nonhyperbolic form $q$ set $N(q)=\operatorname{dim} q-2^{\operatorname{deg} q}$.

We write $X_{F}$ for the set of orderings on $F$. A form $q$ is indefinite at $\alpha \in X_{F}$ if $\left|\operatorname{sgn}_{\alpha} q\right|<\operatorname{dim} q ; q$ is indefinite if it is indefinite at all $\alpha \in X_{F}$. The Hasse number of $F, \tilde{u}(F)$, is defined to be $\sup \{\operatorname{dim} q\}$, where $q$ ranges over all anisotropic indefinite $F$-forms.

\section{Good forms of height two.}

Definition. A form $q$ over $F$ is a good form if the leading form of $q$ is defined over $F$.

Examples of good forms are excellent forms (cf. [13]). We wish to classify height two good forms in several instances. First we summarize Knebusch's work on good forms.

TheOREM 1.1 (KNEBUSCH). Let $q$ be an anisotropic form of height 2 and degree $n \geqslant 1$ such that $1 \in D(q)$. Then:

(1) $q$ is good iff $q \equiv \rho \bmod J_{n+1} F$ for some $n$-fold Pfister form $\rho$.

(2) $q$ is excellent iff $q \simeq a \rho \sigma^{\prime}$, with $a \in \dot{F}, \quad \rho$ an $n$-fold and $\sigma$ an $i$-fold Pfister form, $i \geqslant 2$.

(3) Suppose $q$ is good, but not excellent, with leading form $\rho$. Then $q \otimes F(\rho)$ is anisotropic and lies in $G P_{m} F(\rho)$, with $m \geqslant n+1$; in particular, $\operatorname{dim} q=2^{m}$. Further, the Witt index $i(q-\rho)=2^{j}, j \geqslant 0$, and $q$ and $\rho$ have as a common divisor a $j$-fold Pfister form.

Proof. [13, 9.6, 10.1 and 10.5].

Proposition 1.2. Let $q$ be an anisotropic form of odd dimension. Then the following are equivalent:

(1) $q$ is good of height 2.

(2) $q$ is excellent of height 2.

(3) $q \simeq y\left(\rho \sigma^{\prime} \perp\langle 1\rangle\right)$, where $\rho \in P_{n} F, n \geqslant 2, \sigma \in P F$ and $y=d(q)$. 
Proof. (1) $\Rightarrow(2)$. We have

$$
\operatorname{ker}(q \otimes F(q)) \simeq y \rho^{\prime} \otimes F(q),
$$

for some $\rho \in P_{m} F(m \geqslant 2)$ and $y \in F(q)$. Taking discriminants yields $y \equiv$ $d(q) \bmod \dot{F}(q)^{2}$. Then

$$
\operatorname{ker}(q \otimes F(q)) \simeq d(q) \rho^{\prime} \otimes F(q),
$$

so $q$ is excellent by $[\mathbf{1 3}, 7.14]$.

(2) $\rightarrow$ (3). Let $\tau$ be the associated Pfister form of $q$ and $\alpha$ its complementary form. So, $q \perp \alpha \simeq t \tau$, for some $t \in \dot{F}$, with $\operatorname{dim} \alpha<\frac{1}{2} \operatorname{dim} \tau$ and $\operatorname{dim} \alpha$ odd. Since $q$ is excellent of height 2 , by [13, pp. 3-4] $\alpha$ is excellent of height 1 . Hence, $\alpha \simeq y \rho^{\prime}$ for some $y \in \dot{F}, \rho \in P_{n} F$ and $n \geqslant 2$. Then $\alpha \otimes F(\rho)$ is isotropic, so $\tau$ splits over $F(\rho)$ and so $\rho \mid \tau$. By $[4,1.4], \tau \simeq \rho \sigma$, for some $\sigma \in P F$. From the isometry $q \perp y \rho^{\prime} \simeq t \tau$, we obtain that for any $c \in D\left(\rho^{\prime}\right) \subset D(\tau), y c t \in D(\tau)$. Hence $y t \in D(\tau)$ and

$$
q \perp y \rho^{\prime} \simeq t \tau \simeq y \tau \simeq y\left(\rho \sigma^{\prime} \perp\langle 1\rangle \perp \rho^{\prime}\right) .
$$

Condition (3) follows by cancelling $y \rho^{\prime}$ from each side and computing the discriminant.

$(3) \rightarrow(1)$. Since $q \perp y \rho^{\prime} \simeq y \rho \sigma, q \otimes F(q)=-y \rho^{\prime} \otimes F(q)$, so $q$ is a good form of height 2 .

Throughout the remainder of this section, we assume $q$ is an even-dimensional anisotropic form.

The classification theorem we would like for height 2 good forms is:

(1.3) $q$ is a height 2 good form of degree $n$ if and only if $q$ is isometric to either:

(1) $a \rho \sigma^{\prime}$, with $a \in \dot{F}, \rho \in P_{n} F$ and $\sigma \in P_{m} F$, for some $m \geqslant 2$, or

(2) $\rho q_{1}$, with $\rho \in P_{n-1} F, \operatorname{dim} q_{1}=4$ and $d\left(q_{1}\right) \notin D(\rho)$. Further $q$ is excellent iff $q$ is of type (1).

It is easy to check that forms of types (1) and (2) are good forms of height 2 and degree $n$. The last statement follows from (1.1). Knebusch [13, 10.3] has shown for forms of degree 1 that a height two form is good and (1.3) holds.

LEMMA 1.4. Let $q$ be a anisotropic form and suppose $q=x p \perp q_{1}$, where $x \in \dot{F}$, $\rho \in P_{n} F$ and $\operatorname{deg} q_{1}=m \geqslant n+2$. If $q$ has height 2 then $\rho \mid q$.

Proof. The leading form of $q$ is $\rho$ by $[13,9.5]$. For some $a \in F(q),\langle a,-x\rangle \rho \otimes$ $F(q)=q_{1} \otimes F(q)$. Since $\langle a,-x\rangle \rho$ is similar to a $(n+1)$-fold Pfister form and $\operatorname{deg} q_{1}>n+1$, we must have

$$
\langle a,-x\rangle \rho \otimes F(q)=0 \text { and }(q-x \rho) \otimes F(q)=0 .
$$

Then $q-x \rho$ is similar to an anisotropic Pfister form by [13, 7.13] (cf. also [9, 1.6]). Thus $q \otimes F(\rho)=0$ and $\rho \mid q$.

Following [7] we say a field extension $K / F$ is excellent if for every $F$-form $q$, $\operatorname{ker}(q \otimes K)$ is defined over $F$ (see [7, 2.1] for equivalent definitions).

Proposition 1.5. Let $q$ be an anisotropic form of height 2 that is good but not excellent. Suppose the leading form of $q$ is defined by $\rho \in P_{n} F$. If $F(\rho) / F$ is excellent, then $\operatorname{dim} q=2^{n+1}$. 
Proof. We may assume $1 \in D(q) ; q=x \rho \perp q_{1}$ with $x \in \dot{F}$, and $\operatorname{deg} q_{1} \geqslant n+1$, by (1.1). Suppose $\operatorname{dim} q>2^{n+1}$. Since $q$ is not excellent, by $[13,10.1]$ we have that $q \otimes F(\rho)$ is an anisotropic Pfister form of degree $\geqslant n+2$. By [7, 2.10] there is a $\sigma \in P_{m} F(m \geqslant n+2)$ such that $\sigma \otimes F(\rho) \simeq q \otimes F(\rho)$. Hence, $q=\sigma \perp \rho q_{2}$ with $\operatorname{dim} q_{2}$ odd.

Let $d=d\left(q_{2}\right)$. Then $q_{2} \perp\langle d\rangle=q_{3} \in I^{2} F$. So

$$
q=-d \rho \perp \rho q_{3} \perp \sigma .
$$

Since $\operatorname{deg}\left(\rho q_{3} \perp \sigma\right) \geqslant n+2, \rho \mid q$ by (1.4), which contradicts (1.1).

THEOREM 1.6. The classification (1.3) holds for forms of degree 2.

Proof. Let $q$ be a good, but not excellent, form of height and degree 2. We need only show $q$ is of type (2). But Arason [1] has shown $F(\rho) / F$ is excellent for 2-fold Pfister forms $\rho$. Thus $\operatorname{dim} q=8$ by (1.5) and $q$ is divisible by a binary form by [13, 10.8]. That is, $q \simeq\langle\langle a\rangle\rangle q_{1}$, with $\operatorname{dim} q_{1}=4$. Since $\operatorname{deg} q=2$ we see that $d\left(q_{1}\right) \notin$ $D(\langle\langle a\rangle\rangle)$ as required.

Note that the good forms of height and degree 2 are precisely the forms in $I^{2} F$ having Clifford invariant equal to a quaternion algebra (cf. [13, 9.5]).

We have not been able to classify good forms of height 2 and degree $\geqslant 3$ without restricting the field $F$. We first consider fields with small Hasse number.

LEMMA 1.7. Let $q$ be an anisotropic good, but not excellent, form of height 2 and degree $n \geqslant 1$. Suppose

$$
q \equiv \sigma \perp \underset{i=1}{\perp} x_{i} \rho_{1} \quad \bmod J_{n+2},
$$

where $\sigma \in P_{n} F$, each $x_{i} \in F, \rho_{i} \in P_{n+1} F$ and $k \leqslant 2$. Then $\operatorname{dim} q=2^{n+1}$.

Proof. We need only consider the case $k=2$ (if $k=1$, take $\rho_{2}=2^{n} \cdot\langle 1,-1\rangle$ ). Set $\tau=\operatorname{ker}\left(\rho_{1}-\rho_{2}\right) \in J_{n+1} F$.

Suppose $\operatorname{dim} q>2^{n+1}$. Then $q \otimes F(\sigma)$ is similar to a Pfister form of degree at least $n+2$ (1.1). That is, $\left(x_{1} \rho_{1} \perp x_{2} \rho_{2}\right) \otimes F(\sigma) \in J_{n+2} F(\sigma)$. Since

we have

$$
\begin{gathered}
\operatorname{dim}\left(x_{1} \rho_{1} \perp x_{2} \rho_{2}\right)=2^{n+2}, \\
\left(x_{1} \rho_{1} \perp x_{2} \rho_{2}\right) \otimes F(\sigma) \in G P_{n+2} F(\sigma), \\
\left(x_{1} \rho_{1} \perp x_{2} \rho_{2}\right) \otimes F(\sigma, \rho)=0,
\end{gathered}
$$

so $\rho_{1} \otimes F(\sigma) \simeq \rho_{2} \otimes F(\sigma)$. Hence, $\tau \otimes F(\sigma)$, and then $\tau \simeq \sigma \gamma$ for some form $\gamma$.

Since $\tau \in J_{n+1} F, \operatorname{dim} \gamma$ is even. Then $\operatorname{dim} \gamma=2$ or 0 , as $\operatorname{dim} \tau<2^{n+2}$. Thus

$$
\begin{aligned}
q & \equiv \sigma \cdot\langle a, b, c\rangle & & \bmod J_{n+2} F, \\
& \equiv-a b c \sigma & & \bmod J_{n+2} F .
\end{aligned}
$$

Then $\sigma \mid q$ by (1.4) and $q$ is excellent [13, 10.1], a contradiction.

Proposition 1.8. Suppose $F$ is $S A P$ and $J_{m} F \cap W_{t} F \subset G P_{m} F$. Let $q$ be an anisotropic good, but not excellent, even-dimensional form of height 2 . If $\operatorname{deg} q=n \geqslant$ $m-1$, then

$$
q \simeq p \psi, \text { where } \rho \in P_{n-1} F \text { and } \operatorname{dim} \psi=4
$$


Proof. Write $q=\sigma \perp q_{1}$, with $\sigma \in P_{n} F$ and $\operatorname{deg} q_{1} \geqslant n+1 \geqslant m$; we may assume $1 \in D(q)$. It suffices to show $\operatorname{dim} q=\operatorname{dim} q_{1}=2^{n+1}$ by (1.1).

Claim. It suffices to show there is a $j \geqslant n+1$ with $\left\{\left|\operatorname{sgn}_{\alpha} q_{1}\right|: \alpha \in X_{F}\right\} \subseteq\left\{0,2^{j}\right\}$.

Given such a $j$, we may find $e_{1}, e_{2} \in \dot{F}$ such that $e_{1}>{ }_{\alpha} 0$ if and only if $\operatorname{sgn}_{\alpha} q_{1}<0$, and $e_{2}>{ }_{\alpha} 0$ if and only if $\operatorname{sgn}_{\alpha} q_{1} \neq 0$, since $F$ is SAP (cf. [14]). Set $\tau=2^{j-1}\left\langle e_{1}, e_{1} e_{2}\right\rangle$. Then $q_{1} \perp \tau \in J_{m} F \cap W_{t} F$. By hypothesis, $q_{1} \perp \tau=\phi$ for some $\phi \in G P_{m} F \cup\{0\}$. If $\phi=0$, we are done by (1.4) and (1.1). Otherwise, deg $q_{1} \geqslant n+$ 1 , $\operatorname{deg} \tau \geqslant n+1$ and $n+1 \geqslant m$, so we must have $n+1=m$. Then (1.7) implies $\operatorname{dim} q=2^{n+1}$.

Lastly, $q_{1}$ is the difference of scalar multiples of the two Pfister forms $\phi$ and $\tau$, so $\operatorname{dim} q_{1}=2^{n+1}+2^{j}-2^{i}$, for some $i \leqslant n+1$ (cf. proof of [4, 4.5]). However, by (1.1), $\operatorname{dim} q_{1}=2^{n+1}+2^{n}-2^{l}$, some $l \leqslant n$. We obtain $j=n+1=i$, so $\operatorname{dim} q_{1}=$ $2^{n+1}$.

We now show there is indeed a $j$ such that $\left\{\left|\operatorname{sgn}_{\alpha} q_{1}\right|: \alpha \in X_{F}\right\} \subset\left\{0,2^{j}\right\}$. Let $\operatorname{dim} q=2^{k}$, where $k \geqslant n+1$. Take any $\alpha \in X_{F}$ and let $F_{\alpha}$ be a real closure of $F$ with respect to $\alpha$. We have:

$$
\begin{aligned}
& \operatorname{sgn}_{\alpha} q=\operatorname{sgn}_{\alpha} \sigma+\operatorname{sgn}_{\alpha} q_{1} ; \\
& 2^{n+1} \mid \operatorname{sgn}_{\alpha} q_{1}, \quad \text { as } \operatorname{deg}\left(q_{1} \otimes F_{\alpha}\right) \geqslant \operatorname{deg} q_{1} \geqslant n+1 ; \\
& \left|\operatorname{sgn}_{\alpha} q\right|=0,2^{n} \text { or } 2^{k}, \quad \text { as } h(q)=2 ; \\
& \operatorname{sgn}_{\alpha} \sigma=0 \text { or } 2^{n}, \quad \text { as } \sigma \in P_{n} F .
\end{aligned}
$$

The possibilities for the signatures are thus:

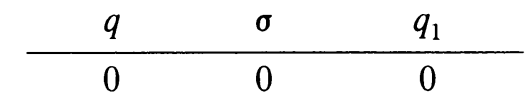

$-2^{n}$

$2^{n}-2^{n+1}$

$2^{n}$

$2^{n}$

0

$\pm 2^{k}$

$\pm 2^{k}$

If both cases (b) and (d) occur, we must show $n=k+1$. (Unless both cases occur, the argument is already finished.) Suppose there are $\beta, \delta \in X_{F}$, with $\beta$ of type (b) and $\delta$ of type (d). Set $K=F_{\beta} \cap F_{\delta}$. Then $K$ is Pythagorean and also SAP, since $\left|X_{K}\right|=2$, as $\left|\dot{K} / \dot{K}^{2}\right| \leqslant 4$. Because both $\beta$ and $\delta$ extend to $K, q \otimes K$ is anisotropic and good, but not excellent, of height 2. Now choose $e_{1}, e_{2} \in \dot{K}$ such that $e_{1}$ is positive only at $\beta$ and $e_{2}$ is positive only at $\delta$. Set $\phi=-2^{n}\left\langle\left\langle e_{1}\right\rangle\right\rangle$ and $\psi=$ $2^{k-1}\left\langle\left\langle e_{2}\right\rangle\right\rangle$, if $\operatorname{sgn}_{\delta} q=2^{k}$, otherwise set $\psi=-2^{k-1}\left\langle\left\langle e_{2}\right\rangle\right\rangle$. Then since $K$ is Pythagorean, $q_{1} \otimes K=\phi \perp \psi$. Hence (1.7) implies $2^{k}=\operatorname{dim} q \otimes K=2^{n+1}$, so $k=$ $n+1$.

THEOREM 1.9. Suppose $F$ is $S A P$ and $J_{4} F \cap W_{t} F \subset G P_{4} F$. Then the classification (1.3) holds for all $q$ of degree $n \geqslant 1$.

Proof. This follows from (1.8) and (1.6).

Fields of Hasse number $\leqslant 16$ satisfy the hypotheses of (1.9), namely if $\tilde{u}(F) \leqslant 16$ then $F$ is 4-linked, hence SAP, [5, 3.5] and any anisotropic $q \in J_{4} F \cap W_{t} F$ has 
dimension 16 and so lies in $G P_{4} F$. Such fields include fields of transcendence degree at most four over $\mathbf{C}$, global, local and finite fields (cf. [3]). However there are fields satisfying the hypothesis of (1.9) with even infinite Hasse number. For example, let $F$ be the power series field $Q((t)) . F$ is SAP since it has only two orderings and $J_{4} F$ is torsion-free since $u(F)=8$, by Springer's Theorem. But clearly $\tilde{u}(F)=\infty$.

We now prove the analogous theorem for fields of transcendence degree at most two over $\mathbf{R}$.

TheOREM 1.10. Suppose $\mathbf{R} \subseteq F$ and tr. $d_{\mathbf{R}} F \leqslant 2$. Then the classification (1.3) holds for all forms $q$ of degree $n \geqslant 1$.

Proof. Let $q$ be a good, not excellent, form of degree $n \geqslant 3$ and height 2 . We need only show $q$ is of type (2) by our previous work. We may assume $q=\rho \perp q_{1}$, with $\rho \in P_{n} F$ and $\operatorname{deg} q_{1} \geqslant n+1$.

Step 1. (a) If $\operatorname{deg} \psi=m \geqslant 3$ then $2^{m-2} \mid \psi$.

(b) Any $\psi \in I^{2} F$ is expressible as $\psi=\alpha \perp \beta \perp \gamma$ with $\alpha, \beta \in G P_{2} F$ and $\gamma \in I^{3} F$.

Using the theorem of Tsen-Lang, one can easily show (a) holds if $\psi$ is an $m$-fold Pfister form. By [2, Satz 17], $J_{m} F=I^{m} F$ is generated by $m$-fold Pfister forms, so (a) holds for all $\psi$ of degree $m$.

To prove (b), let $K=F(\sqrt{-1})$. Then $\psi \otimes K \in I^{2} K=G P_{2} K$, by the theorem of Tsen-Lang. Thus we can find an $\alpha \in G P_{2} F$ such that $\alpha \otimes K=\psi \otimes K[6,2.2]$. So $\psi-\alpha=2 \phi$ for some $\phi \in I F$. Write $\phi=\langle 1,-d(\phi)\rangle \perp \phi_{1}$, where $\phi_{1} \in I^{2} F$; take $\beta=\langle\langle 1,-d(\phi)\rangle\rangle$ and $\gamma=2 \phi_{1}$.

Step 2. $\operatorname{dim} q=2^{n+1}$.

Using both parts of Step 1 we may find $\alpha, \beta \in G P_{2} F$ and $\gamma \in I^{3} F$ such that

$$
\begin{aligned}
q_{1} & =2^{n-1}(\alpha \perp \beta \perp \gamma), \\
q & \equiv \rho \perp 2^{n-1} \alpha \perp 2^{n-1} \beta \bmod J_{n+2} F .
\end{aligned}
$$

Thus $\operatorname{dim} q=2^{n+1}$ by (1.7).

Step 3. There is a $\sigma \in P_{n-1} F$ such that $\sigma \mid q$.

We can write $q \simeq 2^{n-2} \psi$, with $\operatorname{dim} \psi=8$, by Steps 1(a) and 2. By passing to $F(\sqrt{-1})$ and again using the theorem of Tsen-Lang, we obtain $\psi \simeq \psi_{0} \perp 2 \psi_{1}$, with $\operatorname{dim} \psi_{0}=0,2$ or 4 . If $\operatorname{dim} \psi_{0}=0$ then $2^{n-1} \mid q$. If $\operatorname{dim} \psi_{0}=2$ then $\operatorname{dim} \psi_{1}=3$, so $q \otimes F\left(2^{n-2} \psi_{0}\right)=2^{n-1} \psi_{1}$, which has degree $n-1$ unless it is hyperbolic. Thus $2^{n-2} \psi_{0} \mid q$.

Lastly, if $\operatorname{dim} \psi_{0}=4$, then $\operatorname{dim} \psi_{1}=2$. In particular, $2^{n-1} \psi_{1} \in G P_{n} F$. Since $\operatorname{deg} q=n, \operatorname{deg} 2^{n-2} \psi_{0}=n$, so $2^{n-2} \psi_{0} \in G P_{n} F$ also. We need only show $2^{n-1} \psi_{1}$ and $2^{n-2} \psi_{0}$ are linked. We have

$$
q=\rho \perp q_{1}=2^{n-2} \psi_{0} \perp 2^{n-1} \psi_{1}
$$

and

$$
-\rho \perp 2^{n-2} \psi_{0} \perp 2^{n-1} \psi_{1} \in J_{n+1} F .
$$

By $[4,4.8], 2^{n-2} \psi_{0}$ and $2^{n-1} \psi_{1}$ are linked. 
2. Forms of height and degree two. We begin with a lemma due to Knebusch, which is stated, but not proved, in [11]. We include a proof for completeness:

LEMMA 2.1 (KNEBUSCH). Let $\rho$ be an anisotropic Pfister form and $q$ a subform of $\rho$. Then there is a Pfister form $\sigma$ such that $q<\sigma<\rho$ and $\operatorname{deg} \sigma \leqslant \operatorname{dim} q-1$.

Proof. We may assume $1 \in D(q)$. We use induction on $\operatorname{dim} q$. the result is trivially true if $\operatorname{dim} q=1$.

Suppose $q \simeq\left\langle 1, a_{1}, \ldots, a_{i}\right\rangle, i \geqslant 1$. If $\operatorname{deg} \rho \leqslant i$, we are done. Otherwise, by induction, there is a Pfister form $\sigma_{i}$ such that $\left\langle 1, a_{1}, \ldots, a_{i-1}\right\rangle<\sigma_{1}<\rho$, and $\operatorname{deg} \sigma_{i} \leqslant i-1$. Write $q \perp \phi \simeq \rho,\left\langle 1, a_{1}, \ldots, a_{i-1}\right\rangle \perp \psi_{i} \simeq \sigma_{i}$ and $\rho \simeq \sigma_{i} \rho_{i}$, with $\rho_{i} \in$ $P F$. Cancellation yields

$$
\left\langle a_{i}\right\rangle \perp \phi \simeq \psi_{i} \perp \sigma_{i} \rho_{i}^{\prime}
$$

so $a_{i}=c+d$, with $c \in D\left(\psi_{i}\right) \cup\{0\}$ and $d \in D\left(\sigma_{i} \rho_{i}^{\prime}\right) \cup\{0\}$. If $d=0$, then $q<\sigma_{i}$ and we are done. If $d \neq 0$, there is Pfister form $\rho_{i+1}$ such that $p \simeq \sigma_{i}\langle\langle d\rangle\rangle \rho_{i+1}[4$, 2.7]. Set $\sigma=\sigma_{i}\langle\langle d\rangle\rangle$.

The following is a straightforward generalization of $[\mathbf{1 0}, 1.2]([\mathbf{1 0}, 1.2]$ covers the case $\rho=\langle 1\rangle)$.

LEMMA 2.2. Let $\phi$ and $\psi$ be anisotropic forms over $F$ and $\rho \in P F($ or $\rho=\langle 1\rangle)$. Suppose $\phi \simeq \psi \perp x \rho$, for some $x \in \dot{F}$, and $\rho \mid \psi$. If $W(F(\psi) / F)$ is a strong $n$-Pfister ideal then:

(1) $W(F(\phi) / F)$ is $a\{n, n+1\}$-Pfister ideal.

(2) If $\sigma \in W(F(\phi) / F) \cap P_{k} F$, with $k \geqslant n+1$, then there is a $\rho \in W(F(\phi) / F) \cap$ $P_{n+1} F$ such that $\rho \mid \sigma$.

REMARK. The proof is omitted since it requires only slight changes in the proof of $[10,1.2]$. Specifically, in each step of the proof for $[10,1.2]$ where one deduces $\langle b\rangle$ is a subform of $\gamma$ (from $b \in D(\gamma)$ ), one now deduces $b \rho$ is a subform of $\gamma$ (from $b \in D(\gamma)$ and $\rho \mid \gamma)$.

Several other results in [10], namely $1.3-1.5,2.1,2.2$ and 2.4 may be similarly generalized, either as a consequence of 2.2 or by changing their proofs in the same way. We explicitly state one of these generalizations, without proof, since it will be needed later.

LEMMA 2.3 (CF. [10, 1.4]). Let $\psi$ be a neighbor of $\rho \in P_{n} F$ such that $1 \in D(\psi)$. Let $\phi \simeq \psi \perp x \sigma$ be anisotropic, where $x \in \dot{F}, \sigma \in P F($ or $\sigma=\langle 1\rangle)$ and $\sigma \mid \psi$. Then either:

(a) $\phi$ is a neighbor of $\rho$ and $W(F(\phi) / F)=\rho W F$, a strong $n$-Pfister ideal; or

(b) $\phi$ is not a neighbor of $\rho$ and $W(F(\phi) / F)$ is a strong $(n+1)$-Pfister ideal.

REMARK. Lemma 2.3 applies to any form of type (2) in (1.3). Namely, if $\phi \simeq \rho$ $\langle a, b, c, d\rangle$ then take $\psi=\rho \cdot\langle a, b, c\rangle, \sigma=\rho$ and $x=d$.

LEMMA 2.4. Let $\rho_{1}$ and $\rho_{2}$ be $(n-2)$-linked $n$-fold Pfister forms, $n \geqslant 2$. Suppose $q \simeq \rho_{1} \perp x \rho_{2}$, with $x \in \dot{F}$, is an anisotropic form of height 2 . If $W(F(q) / F) \neq 0$ then $W(F(q) / F)$ is a $\{n+2, n+3\}$-Pfister ideal. 
PRoof. Suppose $\rho_{1} \simeq \tau \sigma_{1}, \rho_{2} \simeq \tau \sigma_{2}$, where $\tau \in P_{n-2} F$ and $\sigma_{1}, \sigma_{2} \in P_{2} F$. Write $\sigma_{2}=\langle\langle a, b\rangle\rangle$ and set $\psi=\tau\left(\sigma_{1} \perp x \sigma_{2}^{\prime}\right)$. Since $\tau\left(\sigma_{1} \perp\langle x a\rangle\right)$ is a Pfister neighbor, $W\left(F\left(\tau\left(\sigma_{1} \perp\langle x a, x b\rangle\right)\right) / F\right)$ is a strong $k$-Pfister ideal, $k=n+1$ or $n+2$ by (2.3). Thus $\psi$ is either a Pfister neighbor or $W(F(\psi) / F)$ is a $\{n+2, n+3\}$-Pfister ideal, (2.2).

Now $q \otimes F(\psi)$ is isotropic and also $\psi \otimes F(q)$ is isotropic since

$$
\operatorname{dim}(\operatorname{ker}(\psi \perp x \tau) \otimes F(q))=2^{n}<\operatorname{dim} \psi-\operatorname{dim} \tau .
$$

Thus $F(q)$ is equivalent to $F(\psi)$ over $F$ (in the sense of [12]) and $W(F(q) / F)=$ $W(F(\psi) / F)$.

Lastly, since $\operatorname{dim} q=2^{n+1}$ and $q$ has height 2 , there are no $(n+1)$-fold Pfister forms in $W(F(q) / F)$. Hence $\psi$ is not a Pfister neighbor and $W(F(q) / F)$ is a $\{n+2, n+3\}$-Pfister ideal.

LEMMA 2.5. Let $q$ be an anisotropic form of height and degree 2. Then:

(1) If $\operatorname{dim} q=8$ then $q \simeq a \rho_{1} \perp b \rho_{2}$, with $a, b \in \dot{F}$ and $\rho_{1}, \rho_{2} \in P_{2} F$,

(2) $\operatorname{dim} q \neq 10$.

Proof. Choose an $x$ such that $q \otimes F(\sqrt{-x})$ is isotropic. If $q \otimes F(\sqrt{-x})=0$ then $\operatorname{dim} q \neq 10$ (as $\operatorname{deg} q=2$ ), and if $\operatorname{dim} q=8$ then (1) is clear. So we may assume $q \otimes F(\sqrt{-x}) \neq 0$. Since $h(q)=2$,

$$
q \simeq \phi_{0} \perp\langle 1, x\rangle \phi_{1} \text { and } q \simeq a \rho_{1} \perp q_{1},
$$

with $\operatorname{dim} \phi_{0}=4, \rho_{1} \in P_{2} F$ and $\operatorname{deg} q_{1}=2$.

(1) If $\operatorname{dim} q=8$ then $\operatorname{dim} q_{1}=4$. Thus $q_{1} \in G P_{2} F$ and we are done.

(2) If $\operatorname{dim} q=10$ then $\operatorname{dim} q_{1}=6$. Thus $q_{1}=c \operatorname{ker}\left(\rho_{2}-\rho_{3}\right)$, where $c \in \dot{F}$ and $\rho_{2}$, $\rho_{3}$ are unlinked 2-fold Pfister forms.

Since $\operatorname{dim}\left(\operatorname{ker}\left(q \otimes F\left(\rho_{1}\right)\right)\right)=4$, we have $q_{1} \otimes F\left(\rho_{1}\right)$ isotropic with a kernel similar to a 2-fold Pfister form. By Arason's theorem [1] $F\left(\rho_{2}\right) / F$ is excellent, so there is an $e \in \dot{F}, \rho_{4} \in P_{2} F$ and a form $\psi$ such that

$$
q_{1}=e \rho_{4} \perp \rho_{1} \psi \text { and } q=e \rho_{4} \perp(\psi \perp\langle 1\rangle) .
$$

If $\operatorname{dim} \psi$ is odd then $q$ is good, contradicting (1.6). If $\operatorname{dim} \psi$ is even, then

$$
c \rho_{2}-c \rho_{3}-e \rho_{4}=q_{1}-e \rho_{4} \in I^{3} F .
$$

By $[4,4.8]$ this implies $\rho_{2}$ and $\rho_{3}$ are linked, a contradiction.

Corollary 2.6. If $q$ is an anisotropic form of dimension 10 , then $h(q) \geqslant 3$.

Proof. Clearly $h(q) \geqslant 2$. If $h(q)=2$ we cannot have $\operatorname{deg} q=1$ by $[13,10.3]$ or $\operatorname{deg} q=2$ by (2.5). Since $\operatorname{deg} q \leqslant 2$ by [16, Satz 14], we must have $h(q) \geqslant 3$.

THEOREM 2.7. Let $q$ be an anisotropic form of height and degree 2. Then $q$ is isometric to one of the following:

(1) $a \rho \sigma^{\prime}$, with $a \in \dot{F}, \rho \in P_{2} F$ and $\sigma \in P_{n} F, n \geqslant 2$.

(2) $\langle\langle a\rangle\rangle q_{1}$, with $\operatorname{dim} q_{1}=4, a \in \dot{F}$ and $d\left(q_{1}\right) \notin D(\langle\langle d a\rangle\rangle)$.

(3) $a \cdot \operatorname{ker}\left(\rho_{1}-\rho_{2}\right)$, with $a \in \dot{F}$ and $\rho_{1}, \rho_{2}$ 2-fold Pfister forms, not linked.

(4) $a \rho_{1} \perp b \rho_{2}$, with $a, b \in \dot{F}$ and $\rho_{1}, \rho_{2} 2$-fold Pfister forms, not linked.

(5) $a \cdot \operatorname{ker}(\sigma-\psi)$, with $a \in \dot{F}, \sigma \in P_{5} F$ and $\psi$ a height 2 subform of $\sigma$ of types (3) or (4). 
Proof. If $q$ is a good form, it is of types (1) or (2) by (1.6). Clearly $\operatorname{dim} q \geqslant 6$ and if $\operatorname{dim} q=6$ it is of type (3). If $\operatorname{dim} q=8$, then $q$ is a sum of scalar multiples of two 2-fold Pfister forms by (2.5). Thus $q$ is a type (2) or (4), depending on whether the Pfister forms are linked. We can thus assume $q$ is not excellent and $\operatorname{dim} q>8$.

We may assume $q \simeq\langle 1, x, y, \ldots\rangle$; set $\rho_{2}=\langle\langle x, y\rangle\rangle$. Then $q \otimes F\left(\rho_{2}\right)$ is isotropic, but not zero since then $q$ would be excellent. By Arason's theorem [1], there is $a \in \dot{F}$ and $\rho_{1} \in P_{2} F$ such that

$$
q=a \rho_{1} \perp \rho_{2} \phi, \text { for some } \phi .
$$

Note $\operatorname{dim} \phi$ is odd, since otherwise $q$ is a good form by (1.1).

Write $\phi=\langle b\rangle \perp \phi_{1}$, with $\phi_{1} \in I^{2} F$. Then

$$
q=a \rho_{1} \perp b \rho_{2} \perp \rho_{2} \phi_{1}
$$

and

$$
\operatorname{dim}\left(\operatorname{ker}\left(q-a \rho_{1}-b \rho_{2}\right) \otimes F(q)\right) \leqslant 12 .
$$

Then $\rho_{2} \phi_{1} \in I^{4} F$ implies $\rho_{2} \phi_{1} \otimes F(q)=0$. Now

$$
N\left(\rho_{2} \phi_{1}\right) \leqslant(\operatorname{dim} q+8)-16<\operatorname{dim} q,
$$

so $\rho_{2} \phi_{1} \simeq z \sigma$, with $z \in \dot{F}, \sigma \in P_{n} F$ and $n \geqslant 4$ by $[9,1.6]$.

Since $\sigma \otimes F(q)=0, z^{\prime} \sigma \simeq q \perp \psi$, for some $z^{\prime} \in \dot{F}$ and form $\psi$, by the CasselsPfister theorem. Thus

$$
z^{\prime} \sigma=z \sigma \perp a \rho_{1} \perp b \rho_{2} \perp \psi
$$

and

$$
\left\langle z^{\prime},-z\right\rangle \sigma=a \rho_{1} \perp b \rho_{2} \perp \psi .
$$

The dimension of the right-hand side is $\leqslant \operatorname{dim} \sigma-\operatorname{dim} q+8$, which is less than the dimension of the left-hand side. So

$$
z^{\prime} \sigma \simeq z \sigma, \quad-\psi \simeq \operatorname{ker}\left(a \rho_{1} \perp b \rho_{2}\right)
$$

and

$$
z \sigma \simeq q \perp-\operatorname{ker}\left(a \rho_{1} \perp b \rho_{2}\right) .
$$

Note that $\sigma \neq 0$ since $\operatorname{dim} q>8$. Also $\psi \notin G P F$ since otherwise $\psi \mid q$ and $q$ is excellent.

Claim. $F(\psi) \sim{ }_{F} F(q)$ and $h(\psi)=\operatorname{deg}(\psi)=2$.

Since $\operatorname{dim} \psi \leqslant 8<\operatorname{dim} q, q \otimes F(\psi)$ is isotropic. And $\psi \otimes F(q)$ is isotropic since $\operatorname{dim}(\operatorname{ker}(q \otimes F(q)))=4<\operatorname{dim} \psi$. Thus $(\psi) \sim{ }_{F} F(q)$. Now deg $\psi=2$ since $\operatorname{deg} \sigma \geqslant$ 4 and $\operatorname{deg} q=2$. Lastly, $-\psi \otimes F(\psi)=\operatorname{ker}(q \otimes F(\psi))$ is similar to a Pfister form, hence $h(\psi)=2$.

From our previous work, dim $\psi \leqslant 8$ implies $\psi$ is of types (2), (3) or (4).

Suppose $\psi$ is of type (2). Then for some $c \in \dot{F},\langle\langle c\rangle\rangle \mid \psi$. Since $F(\psi) \sim{ }_{F} F(q)$ we also have $\langle\langle c\rangle\rangle \mid q$. We may write

$$
q \simeq\langle\langle c\rangle\rangle q_{2}, \quad q_{2} \equiv\langle 1, d\rangle \bmod I^{2} F,
$$

and

$$
q \equiv\langle\langle c, d\rangle\rangle \bmod I^{3} F
$$

Thus $q$ is a good form, contradicting (1.6). 
Suppose $\psi$ is of type (3). Recall $n=\operatorname{deg} \sigma$. If $n=4$ then $\operatorname{dim} q=10$, contradicting (2.5). Suppose $n \geqslant 6$. There is a $\sigma_{1} \in P_{5} F$ such that $z \psi<\sigma_{1}<\sigma$ by (2.1). Now $q \otimes F(\sigma)$ is isotropic so $F(q) \sim{ }_{F} F(\psi)$ implies $\psi \otimes F(\sigma)$ is isotropic. Thus $\sigma_{1} \otimes$ $F(\sigma)=0$, a contradiction. Hence $n=5$ as desired.

Suppose $\psi$ is of type (4). If $n=4$ then $\operatorname{dim} q=8$, a contradiction. Suppose $n \geqslant 6$. Clearly $W(F(\psi) / F) \neq 0$. So there is a $\sigma_{1} \in W(F(\psi) / F) \cap P_{i} F, i=4$ or 5 , by (2.4). Again $\psi \otimes F(\sigma)$ isotropic leads to the contradiction that $\sigma_{1} \otimes F(\sigma)=0$. Thus $n=5$ as desired.

Theorem (2.7) is not a complete description of forms of height and degree 2 (but see (2.13)). While any form of types (1), (2) or (3) is a form of height and degree two, the same is not true of types (4) or (5). Indeed there are no known examples of forms of types (4) or (5) which have height two (and it seems unlikely that such examples exist). The construction of a height two form of type (5) would answer in the negative Knebusch's question (8.3) in [13].

However, (2.7) is strong enough for some applications.

COROllary 2.8. If $q$ is an anisotropic form of height and degree 2 which is not excellent, then $\operatorname{dim} q \leqslant 26$.

COROLlary 2.9. If $q$ has height and degree 2 then $W(F(q) / F)$ is either 0 or a Pfister ideal.

Proof. We may assume $q$ is anisotropic (otherwise $F(q)$ is purely transcendental over $F$ by $[12,3.8]$ and $W(F(q) / F)=0)$. We use (2.7). If $q$ is of types (1) or (5) then $q$ is a Pfister neighbor and the result is clear. The result holds for forms of type (4) by (2.4) and type (2) by (2.3). Since forms of type (3) have dimension $6,[10,2.4]$ implies the corollary for this case.

Recall from [10, Remark after 4.1] that if $q$ is not similar to a Pfister form then for each $u \in W(F(q) / F) \operatorname{dim} q \leqslant 2^{\operatorname{deg} u}-2^{\operatorname{deg} q}$. Also if equality holds, then either $q$ is a Pfister neighbor or $W(F(q) / F)$ is not a Pfister ideal. This is of interest as there are no known examples of Witt kernels of forms that are not Pfister ideals.

Using (2.7) we will show that equality cannot hold for $q$ of degree $\leqslant 2$ unless $q$ is a Pfister neighbor.

LEMMA 2.10. Suppose $q$ and $\psi$ forms over $F$, with $\psi \otimes F(q)$ isotropic and $q \otimes F(\psi)$ anisotropic. Then $h(q \otimes F(\psi))=h(q)$.

Proof. Let $L=F(\psi)$. Let $\left\{K_{i} \mid 0 \leqslant i \leqslant h\right\}$ be the standard generic splitting tower of $q$ and let $q_{i}=\operatorname{ker}\left(q \otimes K_{i}\right), 0 \leqslant i \leqslant h$. For $i>0, \psi \otimes K_{i}$ is isotropic (as $K_{i} \supset K_{1}$ $\simeq F(q))$, so $L \cdot K_{i}=K_{i}\left(\psi \otimes K_{i}\right)$ is purely transcendental over $K_{i}[12,3.8]$. Hence $q_{i} \otimes L \cdot K_{i}$ is anisotropic. This is also true by hypothesis if $i=0$, since $K_{0}=F$ and $q_{0}=q$. It thus follows that $h(q \otimes L) \geqslant h(q)$. But the reverse inequality always holds, by $[12,5.13]$.

Proposition 2.11. Let $q$ be an even dimensional anisotropic form not similar to a Pfister form. Suppose $\phi$ is a nonzero form in $W(F(q) / F)$ such that $\operatorname{dim} q=2^{\operatorname{deg} \phi}-$ $2^{\operatorname{deg} q}$. Then $h(q)=2$. 
Proof. We may assume $\phi$ is anisotropic and $1 \in D(q) \cap D(\phi)$. Then $\phi \simeq q \perp \psi$, for some form $\psi$, by the Cassels-Pfister theorem. Note that $\operatorname{deg} \phi \geqslant \operatorname{deg} q+2$ by [2, Satz 18], so $\operatorname{deg} q=\operatorname{deg} \psi$. We use induction on $h(\psi)$.

If $h(\psi)=1$ then $\psi \in G P F$ and $\psi \otimes F(q)=0$ implies $h(q)=2$. So suppose $h(\psi)>1$. Note that $h(\phi)>1$, since otherwise,

$$
\begin{aligned}
\operatorname{dim} \psi & =\operatorname{dim} \phi-\operatorname{dim} q \\
& =2^{\operatorname{deg} \phi}-\left(2^{\operatorname{deg} \phi}-2^{\operatorname{deg} q}\right)=2^{\operatorname{deg} \psi},
\end{aligned}
$$

and $h(\psi)=1$.

Let $K=F(\psi)$ and $\cdot{ }^{\prime}=\operatorname{ker}(\cdot \otimes K)$.

ClaIM. $\operatorname{deg} q^{\prime}=\operatorname{deg} q, \operatorname{dim} q^{\prime}=\operatorname{dim} q$ and $h\left(q^{\prime}\right)=h(q)$.

First, $\operatorname{deg} \phi^{\prime} \geqslant \operatorname{deg} \phi>\operatorname{deg} \psi$. Since $\phi^{\prime}=q^{\prime} \perp \psi^{\prime}$ we have $\operatorname{deg} q^{\prime}=\operatorname{deg} \psi^{\prime}=$ $\operatorname{deg} \psi=\operatorname{deg} q$.

Now suppose $\operatorname{dim} q^{\prime} \neq \operatorname{dim} q$, that is, $q \otimes F(\psi)$ is isotropic. Then there is an $F$-place $F(q) \rightarrow F(\psi) \cup \infty$ and $\phi \otimes F(\psi)=0$. Since $\operatorname{dim} \phi \leqslant 2$. $\max \{\operatorname{dim} q, \operatorname{dim} \psi\},[9,1.6]$ implies $h(\phi)=1$, a contradiction.

Lastly, to show $h\left(q^{\prime}\right)=h(q)$, it suffices to show $\psi \otimes F(q)$ is isotropic by (2.10). We have $-q \otimes F(q)=\psi \otimes F(q)$. If $\psi \otimes F(q)$ is anisotropic we must have $\operatorname{dim} \psi<$ $\operatorname{dim} q$. But then $\operatorname{dim} \phi<2 \cdot \operatorname{dim} q$ and by $[9,1.6], h(\phi)=1$, a contradiction.

Now since $h\left(q^{\prime}\right)=h(q), q^{\prime}$ is not similar to a Pfister form. Clearly $\phi^{\prime} \in$ $W\left(K\left(q^{\prime}\right) / K\right)$. In order to apply induction we need to show $\operatorname{dim} q^{\prime}=2^{\operatorname{deg} \phi^{\prime}}-2^{\operatorname{deg} q^{\prime}}$, which follows from the claim above and the following:

Claim. $\operatorname{deg} \phi=\operatorname{deg} \phi^{\prime}$.

If not, then $\psi$ is a subform of the leading form of $\phi[12,6.11]$. If $L$ is the leading field of $\phi$ then

$$
x \operatorname{ker}(\phi \otimes L) \simeq \psi \otimes L \perp \eta
$$

for some $x \in L$ and form $\eta$ over $L$. Note that $\eta \neq 0$, since otherwise $\operatorname{dim} \phi=2^{\operatorname{deg} \phi}$, $N(\phi)=\operatorname{dim} \phi-2^{\operatorname{deg} \phi}=\operatorname{dim} q$ and $[9,1.6]$ implies $h(\phi)=1$. In particular, $\operatorname{deg} \phi \otimes$ $L<\operatorname{deg} \phi$, so

$$
\operatorname{dim} \eta \geqslant 2^{\operatorname{deg} \eta}=2^{\operatorname{deg} \psi \otimes L} \geqslant 2^{\operatorname{deg} \psi} .
$$

We obtain from $(*)$ :

$$
\begin{aligned}
& 2^{\operatorname{deg} \phi} \geqslant \operatorname{dim} \psi+2^{\operatorname{deg} \psi}, \\
& \operatorname{dim} q+2^{\operatorname{deg} q} \geqslant \operatorname{dim} \psi+2^{\operatorname{deg} \psi}, \\
& \operatorname{dim} q \geqslant \operatorname{dim} \psi .
\end{aligned}
$$

But then $\operatorname{dim} \phi \leqslant 2 \cdot \operatorname{dim} q$ and again $[9,1.6]$ implies $h(\phi)=1$, a contradiction.

We may apply this induction to get $2=h\left(q^{\prime}\right)=h(q)$.

THEOREM 2.12. Let $q$ be an anisotropic form not similar to a Pfister form and suppose $\phi$ is a nonzero form in $W(F(q) / F)$ such that $\operatorname{dim} q=2^{\operatorname{deg} \phi}-2^{\operatorname{deg} q}$. If $\operatorname{deg} q \leqslant 2$ then $q$ is an excellent form of height $\leqslant 2$. In particular, $q$ is a Pfister neighbor. 
Proof. We may assume $\phi$ is anisotropic.

Case $1 . \operatorname{deg} q=0$. Let $L$ be a leading field and $K$ a splitting field of $\phi$. Note that $q \otimes L$ is anisotropic, since otherwise there is an $F$-place $F(q) \rightarrow L \cup \infty$ and $\phi \otimes L=0$. Let $\cdot^{\prime}$ denote $\operatorname{ker}(\cdot \otimes L)$. Then there are $x, y \in L$ such that $x \phi^{\prime} \simeq q^{\prime}$ $\perp\langle y\rangle$, since $\operatorname{dim} q^{\prime}=\operatorname{dim} q=2^{\operatorname{deg} \phi}-1$. Now $y \equiv d(q) \bmod \dot{L}^{2}$, so $x \phi^{\prime}$ is defined over $F$ by, say, $\rho$. Note that $K$ is equivalent to $F(q)$ over $F$. So $\rho \otimes K=\phi^{\prime} \otimes K=0$ implies $\rho \otimes F(q)=0$. Since $\operatorname{dim} \rho=2^{\operatorname{deg} \phi}<2 \operatorname{dim} q, \rho \in G P F$ by $[9,1.6]$, and $q$ is a codimension 1 Pfister neighbor. In particular, $h(q)=1$.

Case 2. $\operatorname{deg} q=1$. In this case $h(q)=2$ by $(2.11)$. Then $q$ is a codimension 2 Pfister neighbor, since otherwise $\operatorname{dim} q=4$, by [13, 10.3], which is impossible. In particular, $q$ is excellent.

Case 3. $\operatorname{deg} q=2$. Again $h(q)=2$ by (2.11). Suppose $q$ is not excellent. Then $\operatorname{dim} q \leqslant 26$ by $(2.8)$ and so $\operatorname{deg} \phi \leqslant 4$. If $\operatorname{deg} \phi=3$, then $q$ is similar to a Pfister form by [2, Satz 18], which contradicts the result that $h(q)=2$. If $\operatorname{deg} \phi=4$ then $\operatorname{dim} q=12$, so $q$ is excellent by $(2.7)$.

Lastly, we point out that forms of height and degree 2 can be determined over some fields.

Proposition 2.13. Suppose $F(\rho) / F$ is excellent for all $\rho \in P_{3} F$. Then an anisotropic form $q$ has height and degree 2 if and only if $q$ is isometric to either:

(1) $a \rho \sigma^{\prime}$, with $a \in \dot{F}, \rho \in P_{2} F$ and $\sigma \in P_{n} F, n \geqslant 2$,

(2) $\langle\langle a\rangle\rangle q_{1}$, with $\operatorname{dim} q_{1}=4, a \in \dot{F}$ and $d\left(q_{1}\right) \notin D(\langle\langle a\rangle\rangle)$, or

(3) $a \cdot \operatorname{ker}\left(p_{1}-p_{2}\right)$, with $a \in \dot{F}$ and $p_{1}, p_{2} 2$-fold Pfister forms, not linked.

Proof. We refer to (2.7); we need only show no form of types (4) or (5) has height 2.

Suppose $q \simeq a \rho_{1} \perp b \rho_{2}$ is a form of type (4). Then $q=a\left(\rho_{1}-\rho_{2}\right) \perp a \sigma$, for $\sigma=\langle 1, a b\rangle p_{2} \in P_{3} F$. Assume $h(q)=2$. Now $q \otimes F(\sigma)$ is isotropic since $\operatorname{dim}\left(\operatorname{ker}\left(p_{1}-p_{2}\right)\right)=6$ and $q \otimes F(\sigma)=a\left(\rho_{1}-\rho_{2}\right) \otimes F(\sigma)$. Since $q$ has height 2 , $\operatorname{ker}(q \otimes F(\sigma))$ is similar to a 2-fold Pfister form. Thus there is a $c \in \dot{F}, \mu \in P_{2} F$ and a form $\psi$ such that $q=c \mu \perp \sigma \psi$ by the hypothesis that $F(\sigma) / F$ is excellent and [7, 2.10]. But then $q$ is a good form by (1.1) and hence of type (2), a contradiction.

Now suppose $q$ is of type (5), that is, $z \sigma \simeq q \perp \psi$, where $\sigma \in P_{5} F$ and $\psi$ is of type (3). Let $\mu \in P_{3} F$ be a divisor of $\sigma$. Then $q \otimes F(\mu)=-\psi \otimes F(\mu)$. Again, since $\operatorname{dim} \psi=6, q \otimes F(\mu)$ is isotropic. The argument above then shows $h(q) \geqslant 3$.

REMARK. The only case where it is known that $F(\rho) / F$ is excellent for some anisotropic $\rho \in P_{n} F, n \geqslant 3$, is where $\tilde{u}(F) \leqslant 4[8,6.5]$. And if $\tilde{u}(F) \leqslant 4$, then $F$ is linked and the conclusion of (2.12) is clear. However, there are no examples known where $F(\rho) / F$ is not excellent for $\rho \in P F$.

ACKNOWLEDGEMENT. I would like to thank Adrian Wadsworth for his many suggestions on how to improve both the form and content of this paper.

\section{REFERENCES}

J. Arason, Excellence of $F(\phi) / F$ for 2-fold Pfister forms, Appendix II of [7].

L. .. Arason and M. Knebusch, Über die Grade quadratischer Formen, Math. Ann. 234 (1978), 167-192.

3. R. Elman, Quadratic forms and the u-invariant. III, Conf. Quadratic Forms, 1976 (G. Orzech, ed.), Queen's Papers on Pure and Appl. Math., No. 46, Queen's Univ., Kingston, Ont., 1977, pp. 422-444. 
4. R. Elman and T. Y. Lam, Pfister forms and K-theory of fields, J. Algebra 23 (1972), 181-213.

5. , Quadratic forms over formally real fields and pythagorean fields, Amer. J. Math. 94 (1972), 1155-1194.

6. Quadratic forms under algebraic extensions, Math. Ann. 219 (1976), $21-42$.

7. R. Elman, T. Y. Lam and A. Wadsworth, Amenable fields and Pfister extensions, Conf. Quadratic Forms, 1976 (G. Orzech, ed.), Queen's Papers on Pure and Appl. Math., No. 46, Queen's Univ. Kingston, Ont., 1977, pp. 445-492.

8. ___ Function fields of Pfister forms, Invent. Math. 51 (1979), 61-75.

9. R. Fitzgerald, Function fields of quadratic forms, Math. Z. 178 (1981), 63-76.

10 , Witt kernels of function field extensions, Pacific J. Math. 109 (1983), 89-106.

11. E. Gentile and D. Shapiro, Conservative quadratic forms, Math. Z. 163 (1978), 15-23.

12. M. Knebusch, Generic splitting of quadratic forms. I, Proc. London Math. Soc. (3) 33 (1976), 65-93.

13. __ Generic splitting of quadratic forms. II, Proc. London Math. Soc. (3) 34 (1977), 1-31.

14. M. Knebusch, A. Rosenberg and R. Ware, Structure of Witt rings, quotients of abelian group rings, and orderings of fields, Buil. Amer. Math. Soc. 77 (1971), 205-210.

15. T. Y. Lam, The algebraic theory of quadratic forms, Benjamin, New York, 1973.

16. A. Pfister, Quadratische Formen in beliebigen Körpern, Invent. Math. 1 (1966), 116-132.

DePartment of MAThematics, SOUTHern Illinois University, CaRbondale, Illinois 62901 